\title{
The revised FLACC observational pain tool: improved reliability and validity for pain assessment in children with cognitive impairment
}

\author{
SHOBHA MALVIYA MD, TERRI VOEPEL-LEWIS MSN RN, \\ CONSTANCE BURKE BSN RN, SANDRA MERKEL MS RN AND \\ ALAN R. TAIT PhD \\ Department of Anesthesiology, University of Michigan Health Systems, Ann Arbor, MI, USA
}

\section{Summary}

Background: Difficulty with pain assessment in individuals who cannot self-report their pain poses a significant barrier to effective pain management. However, available assessment tools lack consistent reliability as pain measures in children with cognitive impairment (CI). This study evaluated the validity and reliability of the revised and individualized Face Legs Activity Cry and Consolability (FLACC) behavioral pain assessment tool in children with CI.

Methods: Children with CI scheduled for elective surgery were studied. The FLACC was revised to include specific descriptors and parent-identified, unique behaviors for individual children. The child's ability to self-report pain was evaluated. Postoperatively, two nurses scored pain using the revised FLACC scale before and after analgesic administration, and, children self-reported a pain score, if able. Observations were videotaped and later viewed by experienced nurses blinded to analgesic administration.

Results: Eighty observations were recorded in 52 children aged 4-19 years. Twenty-one parents added individualized pain behaviors to the revised FLACC. Interrater reliability was supported by excellent intraclass correlation coefficients (ICC, ranging from 0.76 to 0.90 ) and adequate $\kappa$ statistics $(0.44-0.57)$. Criterion validity was supported by the correlations between FLACC, parent, and child scores $(\rho=0.65-$ $0.87 ; P<0.001)$. Construct validity was demonstrated by the decrease in FLACC scores following analgesic administration $(6.1 \pm 2.6$ vs $1.9 \pm 2.7 ; P<0.001)$.

Conclusions: Findings support the reliability and validity of the FLACC as a measure of pain in children with CI.

Keywords: pain assessment; developmentally delayed children; face legs activity cry and consolability pain tool 


\section{Introduction}

Routine pain assessment has been shown to improve pain management for adults and children (1) and is considered essential for optimal care $(2,3)$. However, difficulty with pain assessment in individuals who cannot self-report their pain poses a significant barrier to effective pain management (4). While some individuals with cognitive impairment $(\mathrm{CI})$ are able to identify the presence of pain, others cannot do so and most particularly the moderately to severely impaired, are unable to quantify its severity (5-11). The availability of valid and reliable observational methods to assess pain in this population is therefore imperative to facilitate effective management.

Recent studies have described a set of core pain behaviors in the cognitively impaired (12-15), many of which are similar to those evident in cognitively intact children. Behavioral categories that have been incorporated into existing pediatric scales may, therefore, provide a reasonable framework to facilitate the objective measure of pain in these children. Indeed, five such tools have been tested in small samples of children with CI, and have been shown to have variable validity and reliability (5, 16-18). Breau et al. (16) tested the reliability and validity of the Noncommunicating Children's Pain Checklist - Postoperative Version (NCCPC-PV) in 25 children with severe CI. This tool requires the scoring of each of 27 types of behavior in six subscales from $0=$ not observed at all, to 3 = observed very often. Although ambiguous from their report, it appears that the composite score could range from 0 to 81 . Breau et al. (16) reported fair interrater reliability for each of the subscales with the poorest reliability in the categories of Sociability, and Body and Limbs. The small sample size and its restriction to the severely impaired, however, limit the ability to generalize these findings to other populations of children with CI. Additionally, the length and nature of this checklist diminishes its utility for routine pain assessment in clinical settings. The Wisconsin Children's Hospital Pain Scale (UWCH) was found to have reasonable reliability and validity in 59 preverbal and 15 nonverbal (i.e. CI) children (18). However, the categories and scoring style of this tool may limit its precision, and are incongruent with most other commonly used measures of pain. Lastly, Schade et al. (17) reported acceptable interrater reliability and discriminant validity as well as a high degree of clinical utility for the Nursing Assessment of Pain Intensity (NAPI) in a small sample of children with cerebral palsy.

The Face Legs Activity Cry and Consolability (FLACC) has been found to have reasonable interrater reliability and validity as a measure of pain in children with varying degrees of CI (5). This simple tool contains five categories, each of which are scored from 0 to 2 to provide a total score ranging from 0 to 10 . However, while measures of agreement between observers were found to be acceptable for comparisons in the Face, Cry and Consolability categories, there was low agreement in the Legs and Activity categories, similar to findings of Breau et al. (16) Lower agreement in these subcategories of existing pain tools may be explained, in part, by the presence of underlying motor impairments, including spasticity, which may cloud behavioral observations. Additionally, some cognitively impaired adults and children have been shown to exhibit unusual pain behaviors including atypical facial responses, laughing, clapping of hands, fidgeting, anger, aggressiveness and self-injury (13,19$23)$, that have not been addressed in existing pain tools. Incorporating such behaviors may improve the validity and reliability of existing pain measures, which may in turn improve our ability to adequately treat pain in this population.

The objectives of the present study were: (i) to revise the FLACC pain assessment tool to include behaviors specific to those with $\mathrm{CI}$, and; (ii) to evaluate the validity and reliability of the revised FLACC in children with CI. The hypothesis tested was that the revised FLACC tool is a valid and reliable measure of pain in this population.

\section{Methods}

This study was approved by the Institutional Review Board at the University of Michigan. Prior to recruitment, the FLACC instrument was revised using the following methods. A thorough review of the literature yielded a comprehensive list of pain behaviors common to individuals with $\mathrm{CI}$ including; agitation, verbal outbursts, tremors, shivering, hypertonicity or increased spasticity, breath holding, 
and gasping $(13-15,21,24,25)$. With reference to this list of behaviors, videotaped segments from a previous study (5) were reviewed to identify those most commonly observed in children with CI who exhibited pain following surgery. The categories themselves (i.e. Face, Legs, Activity, Cry, and Consolability) were unchanged in the revised FLACC tool; however, descriptors were added to incorporate those that were most consistently associated with pain in individuals with CI. FLACC revisions focused primarily on the expansion of descriptors in the least reliable categories (i.e. Legs and Activity). Additionally, as individuals with CI may exhibit idiosyncratic baseline as well as pain behaviors $(20,21,23,24)$, an open-ended descriptor under each category was added to permit parents / caregivers to record divergent pain behaviors for such patients. In order to confirm its content validity, the revised instrument (Table 1) was finally reviewed by several physicians and advanced

Table 1

Revised face legs activity cry and consolability tool and a sample description of individual behavior provided by parents (revisions noted in italics)

Individual behavior ${ }^{a}$

Face

$0=$ No particular expression or smile

1 = Occasional grimace/frown; withdrawn

or disinterested; appears sad or worried

2 = Consistent grimace or frown; frequent/

constant quivering chin, clenched jaw; distressed-looking face;

expression of fright or panic

Individualized behavior:

Legs

$0=$ Normal position or relaxed; usual tone $\mathcal{E}$ motion to limbs

$1=$ Uneasy, restless, tense; occasional tremors

2 = Kicking, or legs drawn up; marked increase in spasticity, constant tremors or jerking

Individualized behavior:

Activity

0 = Lying quietly, normal position, moves easily; Regular, rhythmic respirations

1 = Squirming, shifting back and forth, tense or guarded movements; mildly agitated (e.g. head back and forth, aggression); shallow, splinting respirations, intermittent sighs.

2 = Arched, rigid or jerking; severe agitation;

head banging; shivering (not rigors);

breath holding, gasping or sharp intake of breaths, severe splinting

Individualized behavior:

Cry

$0=$ No cry/verbalization

$1=$ Moans or whimpers; occasional complaint; occasional verbal outburst or grunt

2 = Crying steadily, screams or sobs, frequent complaints; repeated outbursts, constant grunting

Individualized behavior:

Consolability

$0=$ Content and relaxed

$1=$ Reassured by occasional touching, hugging or being talked to. Distractible.

2 = Difficult to console or comfort; pushing away caregiver, resisting care or comfort measures

Individualized behavior:
'Pouty' lip; clenched and grinding teeth' eyebrows furrowed; stressed looking; stern face; eyes wide open - looks surprised; blank expression; nonexpressive

Legs and arms drawn to center of body; clonus in left leg with pain; very tense and still; legs tremble.

Grabs at site of pain; nods head; clenches fists, draws up arms; arches neck; arms startle; turns side to side; head shaking; points to where it hurts; clenches fist to face, hits self, slapping; tense, guarded, posturing; thrashes arms; bites palm of hand; holds breath.

States, 'I'm okay' or 'All done'; mouth wide open \& screaming; states 'Owie' or ' $\mathrm{No}^{\prime}$; gasping, screaming; grunts or short responses; whining, whimpering, wailing, shouting; asks for medicine; crying is rare.

Responds to cuddling, holding, parent, stroking, kissing; distant and unresponsive when in pain.

${ }^{a}$ Excerpts from the additional descriptions of the individual child's pain behavior recorded by parents on the revised Face Legs Activity Cry and Consolability (FLACC) tool during the preoperative interview. Only 21 parents added such comments to the revised FLACC. 
practice nurses who are experts in pain assessment and treatment and in assessment of children with CI via their daily clinical duties and/or work on the pediatric pain service.

With parental consent and child assent, when applicable, children with CI aged 4-21 years of age scheduled for elective surgery were included. Preoperatively, demographic data were recorded and parents were interviewed regarding details of the child's developmental level and communication skills. The child's level of impairment was grossly estimated from parent interview, and by calculating a Deviation IQ Score (DIQ) as: [(developmental age estimate/chronological age) $\times 100$ ], which was categorized as mild, moderate or severe in accordance with the American Psychiatric Association's DSM-IV guidelines for diagnosing mental retardation (26). Additionally, children were evaluated for the presence, extent, and nature of spasticity, mobility, and movement disorders. More specifically, children were tested for their ability to communicate and self-report their pain using a technique described previously $(5,6)$. This testing determined the child's ability to understand magnitude and order using a series of blocks, numbers, and a Simplified Faces Scale (Figure 1). Children who completed all tests were asked how they would prefer to score their pain during the postoperative period (i.e. using either the verbal 0-10 Numbers Scale; the Simplified Faces Scale; or a Simple Word Scale - 'little, medium, or big'). Lastly, parents were asked to review the FLACC pain instrument, and to record additional behavior that may better indicate acute pain in their child (i.e. behavior they have observed during painful episodes). These types of behavior, as well as notations regarding baseline spasticity and mobility, were incorporated into an 'individualized' FLACC tool that was used for all subsequent assessments of the child.

Following emergence from general anesthesia and prior to the administration of analgesics, patients

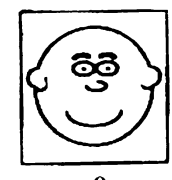

0

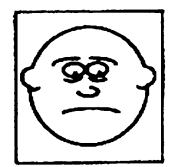

1

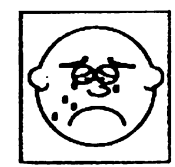

2
Figure 1

Simplified faces scale. were observed and scored for pain behavior using the revised and individualized FLACC pain tool. All observations were made when the child was awake, and most when in the presence of a parent/guardian. Observations were made either in the recovery area or on the general care units. Two nurses trained in the use of the modified FLACC simultaneously but independently observed and recorded pain scores. In addition, parents simultaneously yet independently recorded a global rating of their perception of the child's pain using a $0-10 \mathrm{~cm}$ VAS $(0=$ no pain; $10=$ worst possible pain). For those children deemed able to self-report, a pain score was obtained using either the $0-10$ numbers scale, the simplified faces scale, or a simple word scale (i.e. 'small, medium, big' pain), based on the child's predetermined abilities and preference. Children were videotaped throughout each observation. Parents and/or caregivers were encouraged to interact with and console the child, but did not discuss pain scores or assessment during the taping. Analgesics were given as needed at the discretion of the care providers, and, for those children requiring treatment, observations were repeated in the same manner at $30 \mathrm{~min}$ following analgesic administration.

On completion of recruitment, videotaped segments were randomly mixed, and later viewed by four nurses expert in pediatric pain assessment, who were blinded to the administration of analgesics and to all other assigned pain scores. These nurses assigned pain scores independently using the modified, individualized FLACC tool for half of the segments, and the NAPI (17) for the other half. This tool was chosen over others because of its similarity in structure and ease of use in the clinical setting. Two of these independent observers viewed and scored twenty randomly selected segments each on a second occasion, 3-4 weeks after the first viewing to evaluate test-retest reliability.

Spearman's $\rho$ and ICC were used to determine the strength of association and measure of chancecorrected agreement between scores. The bias between parent and nurse scores was determined by subtracting the bedside nurse and observer scores from parent scores and are presented as mean (bias) and SD (precision). Exact agreement between FLACC scores was determined using \% agreement with kappa statistics $(\kappa)$, where applicable. Scores before 
and after analgesic administration were compared using Wilcoxon signed rank tests for paired data. Correlations $\geq 0.6$ were considered good to excellent associations; $\kappa$ values $\geq 0.41$ were considered adequate agreement; and $P<0.05$ was accepted as statistically significant.

The sample size was based on the following clinical and statistical assumptions: 25 observations would be needed to reveal a correlation of 0.6 $(\alpha=0.05 ; \beta=0.1$ ), which was considered acceptable for the FLACC subcategory reliability comparisons. As this study sought to demonstrate reliability of the FLACC at varying levels of pain, i.e. mild, moderate, and severe, 25 observations were required at each pain intensity (i.e. 75 observations in all). Based on the sample obtained in our previous study (5), it was estimated that approximately 60 children would need to be recruited to ensure at least this number of observations. This sample size was determined to be sufficiently large to satisfy the more robust correlations and comparisons required for interrater, testretest, criterion and construct validity.

\section{Results}

Eighty observations from 52 children were obtained for this study (Table 2). Twelve children successfully completed the tasks necessary to self-report their

Table 2

Description of the study sample $(n=52)$

\begin{tabular}{ll}
\hline Age & $11.3 \pm 4.7$ \\
Gender (female, \%) & $20(39)$ \\
Surgical procedures & \\
Orthopedic & $29(56)$ \\
Oral surgery & $8(15)$ \\
Otologic & $6(12)$ \\
General surgery/other & $9(17)$ \\
Diagnosis & \\
Cerebral palsy & $26(51)$ \\
Syndrome with CI & $9(18)$ \\
Autism & $8(16)$ \\
Other & $8(15)$ \\
Degree of impairment & \\
Mild (DIQ $=82 \pm 5.9)$ & $16(31)$ \\
Moderate (DIQ $=45.3 \pm 12.5)$ & $12(23)$ \\
Severe (DIQ $=11.3 \pm 5.6)$ & $24(46)$ \\
Spasticity & $31(61)$ \\
Quadriplegia & $25(49)$ \\
Hemiplegia & $4(8)$ \\
Diplegia & $2(4)$ \\
\hline
\end{tabular}

DIQ, Deviation IQ Score. pain intensity and chose to use either the Simplified Faces Scale $(n=4), 0-10$ Numbers scale $(n=3)$, or a Simple Word Scale (Little Hurt, Medium Hurt, Big Hurt; $n=5$ ). Most parents confirmed that behavioral descriptors in the FLACC tool were representative of pain behavior in their child; however, 21 parents identified additional types of behavior that were indicative of pain in their children (Table 1).

The interrater reliability of the FLACC was supported by the excellent ICCs and acceptable measures of exact agreement between observers for each of the categories as well as for the total score (Table 3). FLACC scores were coded as mild (scores $0-3)$, moderate (4-6) and severe (7-10), based on previously defined clinically significant pain categories (27-29), to examine reliability for clinically relevant scores; and, analyses demonstrated good to excellent interrater agreement for these scores. To determine whether the reliability of the revised FLACC tool is lower in children with spasticity, agreement was compared between those with and without spasticity for the motor components of the FLACC. Exact agreement was $42-84 \% \quad(\kappa=0.41)$ and $55-74 \%(\kappa=0.41)$ for the Legs and Activity categories, respectively, in children with spasticity $(n=118) ; \quad$ vs $43-87 \% \quad(\kappa=0.49) \quad$ and $\quad 33-89 \%$ $(\kappa=0.53)$ for those without spasticity $(n=88)$. Test-retest reliability was supported by the excellent ICC $\quad(n=32 ; \quad$ ICC $=0.97 ; \quad$ C.I. $=0.92-0.99) \quad$ for repeated FLACC scores of the independent video observers.

Criterion validity was supported by moderate to high correlations between observers' FLACC scores,

Table 3

Interrater reliability between all nurse observers ${ }^{a}$

\begin{tabular}{lcc}
\hline & ICC $^{\text {(confidence interval) }}{ }^{b}$ & \% agreement $(\kappa)^{(\kappa)}$ \\
\hline Face & $0.86(0.83-0.89)$ & $53-81(0.57)$ \\
Legs & $0.77(0.70-0.83)$ & $46-86(0.44)$ \\
Activity & $0.75(0.68-0.80)$ & $50-78(0.45)$ \\
Cry & $0.87(0.83-0.89)$ & $46-89(0.55)$ \\
Consolability & $0.76(0.70-0.81)$ & $64-82(0.48)$ \\
Total score & $0.90(0.87-0.92)$ & NA \\
Coded pain scores $^{c}$ & $0.83(0.78-0.86)$ & $35-89(0.50)$ \\
\hline
\end{tabular}

ICC, intraclass correlation coefficients

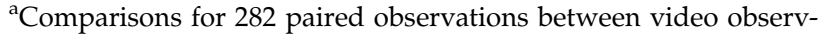
ers, the bedside nurse and the bedside observer.

${ }^{\mathrm{b}}$ All correlations significant at $P<0.001$

'Scores were coded as mild (0-3), moderate (4-6) and severe (7-10) for these comparisons. 
Table 4

Correlations between the nurses' and observers' Face Legs Activity Cry and Consolability (FLACC) and nursing assessment of pain intensity (NAPI) scores, Parent Global Scores, and child ratings

\begin{tabular}{lccc}
\hline & $\begin{array}{c}\text { Parent } \\
(\mathrm{n}=66)\end{array}$ & $\begin{array}{c}\text { Child }^{a} \\
(\mathrm{n}=12)\end{array}$ & $\begin{array}{c}\text { Video observer } \\
\text { NAPI }(\mathrm{n}=77)\end{array}$ \\
\hline Nurse FLACC $(n=80)$ & $0.74^{\mathrm{b}}$ & $0.78^{\mathrm{b}}$ & $0.78^{\mathrm{b}}$ \\
$\begin{array}{c}\text { Bedside observer } \\
\text { FLACC }(n=80)\end{array}$ & $0.82^{\mathrm{b}}$ & $0.86^{\mathrm{b}}$ & $0.87^{\mathrm{b}}$ \\
$\begin{array}{l}\text { Video observer } \\
\text { FLACC }(n=77)\end{array}$ & $0.65^{\mathrm{b}}$ & $0.67^{\mathrm{c}}$ & $0.78^{\mathrm{b}}$ \\
\hline
\end{tabular}

Samples variable based on parent availability, child ability to score pain, and quality of the video segments.

${ }^{\text {a }}$ Scores were coded as mild (0-3), moderate (4-6) and severe (7-10) for these comparisons.

${ }^{\mathrm{b}} P<0.01 ;{ }^{\mathrm{c}} P=0.051$

the parents' global pain ratings, the NAPI scores, and the available child ratings (Table 4). Parent scores tended to be higher than nurse and observer FLACC scores (bias $=1.4$; precision $=2.2$ ), as well as video observer FLACC scores (bias $=1.97$; precision $=2.7$ ). FLACC scores significantly decreased following analgesic administration for both the bedside nurses' assigned scores $(n=20 ; 6.1 \pm 2.5$ vs $2.2 \pm 2.4 ; \quad P<0.001)$ and the blinded video observers' scores $(n=20 ; 6.1 \pm 2.6$ vs $1.9 \pm 2.7$; $P<0.001$ ), supporting the construct validity of the revised tool. Additionally, parent-assigned pain scores similarly decreased $(n=12 ; 6.6 \pm 2.4$ vs $2.6 \pm 2 ; P=0.003)$.

\section{Discussion}

Effective pain management for children with $\mathrm{CI}$ is dependent upon the ability of care providers to reliably observe and assess the presence and intensity of pain. Yet, as recent investigators have pointed out, no standard measure of pain exists for this population (30). Pain tools that have been previously tested in this population are either lengthy or difficult to incorporate into routine care or lack consistent reliability. This study demonstrated that the revised and individualized FLACC tool is a reliable and valid measure of pain in children with CI. This tool offers clinicians an objective method of pain assessment, which may in turn reduce the under-treatment of pain in this vulnerable population.
The FLACC tool was originally designed as a simple observational tool to assess pain in young children who could not self-report their pain (31). The tool is structured around five categories that have been shown to be reliable indicators of pain in children (27). Many of the types of behavior described in the tool have, additionally, been shown to be reliable and sensitive pain indicators in individuals with CI $(12,15)$. These original behavioral descriptors, although not comprehensive, were meant to provide clinicians guidance in making observations and scoring pain intensity. A previous study in children with $\mathrm{CI}$ demonstrated that the Legs and Activity categories of the original FLACC were less reliable than other categories, suggesting that some individuals with CI exhibit a different constellation of pain behavior compared with healthy, cognitively intact children (5). Similar findings were demonstrated by Breau et al. (16) who found that Body, Limbs and Social categories in their pain tool were less reliable, and by Terstegen et al. (12) who showed that facial expressions were more sensitive indicators of pain compared with motor behavior. The revised FLACC tool incorporates several additional behavioral descriptors, including: verbal outbursts, tremors, increased spasticity, jerking movements, and respiratory pattern changes such as breath holding and grunting. This study demonstrates an improvement in reliability measures for all categories as well as for total FLACC scores and coded scores, when compared with our previous study. These findings suggest that the addition of specific behavioral descriptors under the relevant FLACC categories improved the reliability of pain assessment for these children.

In addition to expanded behavioral descriptors, the revised FLACC allows for individualization of the tool via open-ended descriptors in each category. Previous studies have described unique pain behavior in cognitively impaired elderly adults and children, including verbal outbursts and perseverant verbalizations (13), aggression or agitation $(19,20)$, and self-injurious behavior $(21,23,32)$. Additionally, based on their clinical experience Solodiuk and Curley (33) recommended the incorporation of parent-identified descriptors into a commonly used numeric scale to facilitate pain assessment for impaired children. Nearly half of the children in our study were described by their parents as having 
unique behavior indicative of pain. These included: unique facial expressions, leg and body activity including self-stimulating behaviors, specific verbalizations and consoling techniques. Interestingly, several parents noted that a lack of expression, crying, or responsiveness was most indicative of pain in their children. Inclusion of these types of behavior may have contributed to the improved reliability and validity of FLACC pain scores in this study. These findings suggest that for children with $\mathrm{CI}$, a thorough preoperative interview may help to establish baseline and individual behavior that facilitate accurate pain assessment during the postoperative period.

This study evaluated the revised FLACC tool only in children with acute postoperative pain, potentially limiting our ability to generalize its use to other settings. However, studies have identified pain behavior in children with pain related to injury, chronic conditions, illness and medical procedures $(23,34,35)$ that are similar to behavioral descriptors in the FLACC, attesting to its content validity in assessing nonsurgical pain. Additionally, the original FLACC tool was validated in young children with pain of varying etiology, further supporting its use in nonsurgical populations $(36,37)$. The difficulty in scoring individualized behaviors might also be considered to be a limitation. However, the purpose of incorporating such behavior is to provide guidance to clinicians in assessment of children who may exhibit unique behavior. Lastly, our data support the added reliability of the FLACC tool when these types of behavior were included.

The FLACC observational pain assessment tool has been widely used to measure pain intensity in young children who cannot self-report a pain score. This study, in conjunction with prior work, suggests that the FLACC tool provides a reliable and a valid framework for the assessment of pain in children with CI. The addition of specific behavioral descriptors, as well as the incorporation of parent-identified, individualized behavior, improved the psychometric properties of the FLACC tool toward improved pain assessment in this population of children. The tool's simplicity may further facilitate easy assimilation into clinical practice, which may in turn improve clinical outcomes for children with CI.

\section{Conflict of interest statement}

This work does not present any conflict of interest nor bias for any of the authors.

\section{Acknowledgements}

This study was supported by the NIH Grant 5 RO3 HD043920-02. The authors would like to thank Sarah Earle, BS, Julie Conley, BS, and Melissa Doettl, BS for their assistance with this project.

\section{References}

1 Faries JE, Mills DS, Goldsmith KW et al. Systematic pain records and their impact on pain control. A pilot study. Cancer Nurs 1991; 14: 306-313.

2 Joint Commission on Accreditation of Healthcare Organizations Pain Standards for 2001. In: Comprehensive Accreditation Manual for Hospitals: The Official Handbook. Dakbrook, IL: Joint Commission Resources, Inc., 2001.

3 Agency for Health Care Policy and Research. Acute Pain Management: Operative or Medical Procedures and Trauma. Rockville, MD: Department of Health and Human Services Public Health Service, 1992.

4 Malviya S, Voepel-Lewis T, Merkel S et al. Difficult pain assessment and lack of clinician knowledge are ongoing barriers to effective pain management in children with cognitive impairment. Acute Pain 2005; 7: 27-32.

5 Voepel-Lewis T, Merkel S, Tait AR et al. The reliability and validity of the face, legs, activity, cry, consolability observational tool as a measure of pain in children with cognitive impairment. Anesth Analg 2002; 95: 1224-1229.

6 Fanurik D, Koh JL, Harrison RD et al. Pain assessment in children with cognitive impairment. An exploration of selfreport skills. Clin Nurs Res 1998; 7: 103-119.

7 Ferrell BA, Ferrell BR, Rivera L. Pain in cognitively impaired nursing home patients. J Pain Symptom Manage 1995; 10: 591598.

8 Wynne CF, Ling SM, Remsburg R. Comparison of pain assessment instruments in cognitively intact and cognitively impaired nursing home residents. Geriatr Nurs 2000; 21: 20-23.

9 Parmelee PA. Pain in cognitively impaired older persons. Clin Geriatr Med 1996; 12: 473-487.

10 Manz BD, Mosier R, Nusser-Gerlach MA et al. Pain assessment in the cognitively impaired and unimpaired elderly. Pain Manag Nurs 2000; 1: 106-115.

11 Chibnall JT, Tait RC. Pain assessment in cognitively impaired and unimpaired older adults: a comparison of four scales. Pain 2001; 92: 173-186.

12 Terstegen C, Koot HM, de Boer JB et al. Measuring pain in children with cognitive impairment: pain response to surgical procedures. Pain 2003; 103: 187-198.

13 Kovach CR, Weissman DE, Griffie J et al. Assessment and treatment of discomfort for people with late-stage dementia. J Pain Symptom Manage 1999; 18: 412-419.

14 Hadjistavropoulos T, LaChapelle DL, MacLeod FK et al. Measuring movement-exacerbated pain in cognitively impaired frail elders. Clin J Pain 2000; 16: 54-63. 
15 Breau LM, McGrath PJ, Camfield C et al. Preliminary validation of an observational pain checklist for persons with cognitive impairments and inability to communicate verbally. Dev Med Child Neurol 2000; 42: 609-616.

16 Breau LM, Finley GA, McGrath PJ et al. Validation of the noncommunicating children's pain checklist- postoperative version. Anesthesiology 2002; 96: 528-535.

17 Schade JG, Joyce BA, Gerkensmeyer J et al. Comparison of three preverbal scales for postoperative pain assessment in a diverse pediatric sample. J Pain Symptom Manage 1996; 12: 348359.

18 Soetenga D. Assessment of the validity and reliability of the university of Wisconsin children's hospital pain scale for preverbal and nonverbal children. Pediatr Nurs 1999; 25: 670676.

19 Feldt KS, Warne MA, Ryden MB. Examining pain in aggressive cognitively impaired older adults. J Gerontol Nurs 1998; 24: $14-22$.

20 Buffum MD, Miaskowski C, Sands L et al. A pilot study of the relationship between discomfort and agitation in patients with dementia. Geriatr Nurs 2001; 22: 80-85.

21 Fanurik D, Koh JL, Schmitz ML et al. Children with cognitive impairment: parent report of pain and coping. J Dev Behav Pediatr 1999; 20: 228-234.

22 Oberlander TF, Gilbert CA, Chambers CT et al. Biobehavioral responses to acute pain in adolescents with a significant neurologic impairment. Clin J Pain 1999; 15: 201-209.

23 Stallard P, Williams L, Velleman R et al. The development and evaluation of the pain indicator for communicatively impaired children (PICIC). Pain 2002; 98: 145-149.

24 Kovach CR, Noonan PE, Griffie J et al. Use of the assessment of discomfort in dementia protocol. Appl Nurs Res 2001; 14: 193200.

25 Cohen-Mansfield J, Creedon M. Nursing staff members' perceptions of pain indicators in persons with severe dementia. Clin J Pain 2002; 18: 64-73.

26 American Psychiatric Association. (ed.) Diagnostic and Statistical Manual of Mental Disorder, 4th edn. Washington, DC: American Psychiatric Association, 1994.
27 Buttner W, Finke W. Analysis of behavioural and physiological parameters for the assessment of postoperative analgesic demand in newborns, infants and young children: a comprehensive report on seven consecutive studies. Paediatr Anaesth 2000; 10: 303-318.

28 Bodian CA, Freedman G, Hossain S et al. The visual analog scale for pain: clinical significance in postoperative patients. Anesthesiology 2001; 95: 1356-1361.

29 Gauthier JC, Finley GA, McGrath PJ. Children's self-report of postoperative pain intensity and treatment threshold: determining the adequacy of medication. Clin J Pain 1998; 14: 116-120.

30 Koh JL, Fanurik D, Harrison RD et al. Analgesia following surgery in children with and without cognitive impairment. Pain 2004; 111: 239-244.

31 Merkel SI, Voepel-Lewis T, Shayevitz JR et al. The FLACC: a behavioral scale for scoring postoperative pain in young children. Pediatr Nurs 1997; 23: 293-297.

32 Breau LM, Camfield CS, Symons FJ et al. Relation between pain and self-injurious behavior in nonverbal children with severe cognitive impairments. J Pediatr 2003; 142: 498-503.

33 Solodiuk J, Curley MA. Pain assessment in nonverbal children with severe cognitive impairments: the Individualized Numeric Rating Scale (INRS). J Pediatr Nurs 2003; 18: 295-299.

34 Breau LM, Camfield C, McGrath PJ et al. Measuring pain accurately in children with cognitive impairments: refinement of a caregiver scale. J Pediatr 2001; 138: 721-727.

35 Breau LM, McGrath PJ, Camfield CS et al. Psychometric properties of the non-communicating children's pain checklistrevised. Pain 2002; 99: 349-357.

36 Manworren RC, Hynan LS. Clinical validation of FLACC: preverbal patient pain scale. Pediatr Nurs 2003; 29: 140-146.

37 Willis MH, Merkel SI, Voepel-Lewis T et al. FLACC Behavioral Pain Assessment Scale: a comparison with the child's selfreport. Pediatr Nurs 2003; 29: 195-198.

Accepted 5 August 2005 